\title{
The Empirical Properties of Some Popular Estimators of Long Memory Processes
}

\author{
W. Rea ${ }^{\mathrm{a}}$, L. Oxley ${ }^{\mathrm{a}} \underline{\text { M. Reale }}^{\mathrm{b}}{ }^{\text {, J. Brown }}{ }^{\mathrm{b}}$ \\ ${ }^{a}$ Department of Economics and Finance, University of Canterbury, Private Bag 4800, Christchurch, \\ New Zealand \\ ${ }^{\mathrm{b}}$ Department of Mathematics and Statistics, University of Canterbury, Private Bag 4800, Christchurch, \\ New Zealand \\ Email: marco.reale@canterbury.ac.nz
}

\begin{abstract}
The empirical properties of 12 different estimators of the Hurst parameter, $H$, or fractional integration parameter, $d$, derived via simulation, are presented. For time series with fewer than 4,000 observations only the Whittle and Haslett-Raftery estimators produce acceptable statistical properties.
\end{abstract}

Keywords: Strong dependence, global dependence, long range dependence, long memory, Hurst parameter estimators 


\section{INTRODUCTION}

The subject of long-memory time series has received extensive attention in both the statistical and econometric literature particularly financial econometrics. See Beran (1994), Robinson (2003) and Palma (2007).

Of critical importance in modeling long memory time series is estimating the strength of the long-range dependence. Two measures are commonly used. The parameter $H$, known as the Hurst or self-similarity parameter, was introduced by Mandelbrot and van Ness (1968). The fractional integration parameter, $d$, arises from the generalization of the Box-Jenkins ARIMA(p,d,q) models from integer to non-integer values of the integration parameter $d$. This generalization was accomplished independently by Granger and Joyeux (1980) and by Hosking (1981). The parameters $H$ and $d$ are related by the formula $H=$ $d+1 / 2$.

A number of estimators of $H$ and $d$ have been developed and theoretical results produced. Palma (2007, Chapters 4 and 5) gives some details on their asymptotic properties and further references to the literature.

While the asymptotic properties have been established for a number of these estimators, their properties may be quite different in finite series of lengths typical in empirical economic and financial research.

Taqqu et al. (1995) studied nine estimators for a single series length of 10,000 data points, five values of both $H$ and $d$, and 50 replications. Jeong et al. (2007) compared six estimators on simulated series with $32,768\left(2^{15}\right)$ observations, five values of $H$ and 100 replications.

In the results reported here we extend these studies to include a larger number of parameters, higher number of replications and 12 estimators as detailed in Section (2) below.

The remainder of the paper is organized as follows. Section (2) gives details of the method. Section (3) presents the results. Section (4) gives our conclusions.

\section{METHOD}

Ten estimators are implemented in the package fSeries of Wuertz (2005) for the statistical software R (R Development Core Team, 2005). They are the absolute value, aggregated variance, boxed periodogram, differenced variance, Higuchi, Peng, periodogram, rescaled range, wavelet, and the Whittle. Further, the GPH (Geweke and Porter-Hudak, 1983) and Haslett and Raftery (1989) estimators for $d$ is implemented in the contributed package fracdiff of Fraley et al. (2006).

Taqqu et al. (1995) simulated both fractional Gaussian noises (FGNs) and the corresponding discrete time fractionally integrated $(\mathrm{FI}(\mathrm{d}))$ series and found that each estimator performed similarly whether estimating $H$ in simulated FGNs or $d$ in simulated FI(d)s. For example, if an estimator was biased when estimating $H$ it was also biased in a very similar manner when estimating $d$. Thus, for the 10 estimators implemented in fSeries we only investigated each estimator's performance in estimating $H$ for simulated FGNs generated using the function fgnSim in fSeries. For the GPH and HaslettRaftery estimators of $d$ we generated FI(d) series with the function farimaSim in fSeries. We ran 1000 replications of simulated FGNs with 100 different lengths and eight different $H$ values. The lengths were between 100 and 10,000 data points in steps of 100. The $H$ values were between 0.55 and 0.90 , or the equivalent $d$ values, in steps of 0.05 . For each series $H$ was estimated by the ten estimators implemented in fSeries and $d$ by the two estimators implemented in fracdiff. For each $H$ value and series length we estimated the median, $75 \%$ and $95 \%$ confidence intervals empirically from the simulated data. The $H$ or $d$ estimates were sorted into ascending order and the median obtained by averaging the 500th and 501st values. Similar calculations were done for the upper and lower values of the $75 \%$ and $95 \%$ confidence intervals.

In the presentation of the results we converted the GPH and Haslett-Raftery $d$ estimates to $H$ equivalents to facilitate comparisons among the estimators.

\section{RESULTS}

For reasons of space we only present a few representative results. Figures (1) through (6) are presented with the vertical axis with a range of $1.2 \mathrm{H}$ units to facilitate comparisons among the estimators' standard 
deviation of their estimates. It should be noted that stationary long memory occurs in the range $0.5<$ $H<1.0$.

(a) H Est and Cl Absval $\mathrm{H}=0.60$

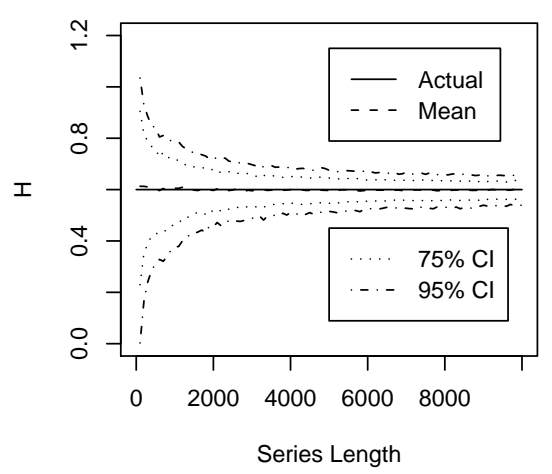

(c) H Est and $\mathrm{Cl}$ Absval $\mathrm{H}=0.90$

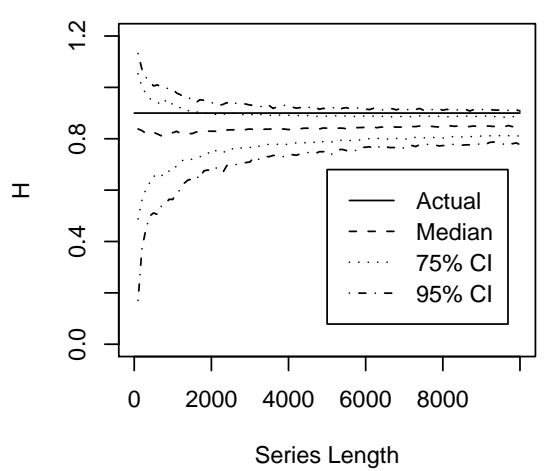

(b) $\mathrm{H}$ Est and $\mathrm{Cl}$ Aggvar $\mathrm{H}=\mathbf{0 . 6 0}$

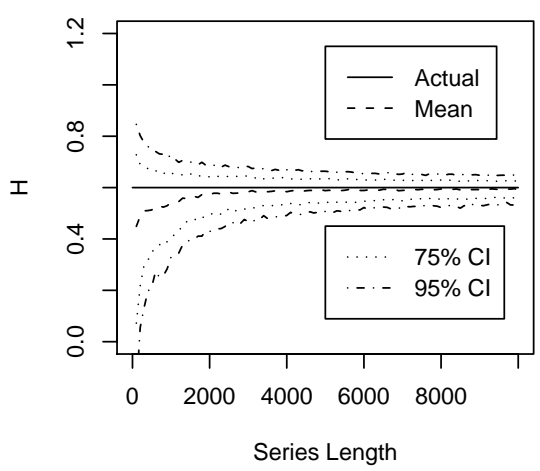

(d) $\mathrm{H}$ Est and $\mathrm{Cl}$ Aggvar $\mathrm{H}=0.90$

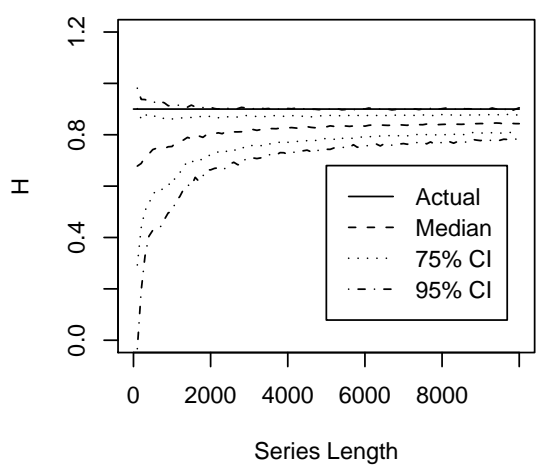

Figure 1. Empirical confidence intervals for the $H$ estimates with $H=0.60$ and $H=0.90$; (a) and (c) absolute value method, (b) and (d) aggregated variance estimator.

The results for the absolute value of the variance method are presented in Figures (1) (a) and (c). It was unbiased at all series lengths when $H$ was low (0.55 or 0.60) but became progressively biased and underestimated $H$ as $H$ increased.

The results for the aggregated variance method are presented in Figures (1) (b) and (d). It exhibited bias and underestimated $H$ in short series when $H$ was low. As $H$ increased the estimator became increasingly biased at all series lengths examined. With $H=0.90$ the true value of $H$ lay above the upper $95 \%$ empirical confidence interval for all but the shortest series lengths.

The results for the boxed periodogram method are presented in Figures (2) (a) and (c). This estimator was developed specifically to deal with perceived problems with the periodogram estimator. It was biased towards underestimating $H$ for almost all values of $H$ and series lengths examined.

The results for the differenced variance method are presented in Figures (2) (b) and (d). This estimator had one of largest confidence intervals of the estimators when the series were short but this slowly decreased as sample size increased. The difference variance estimator exhibited bias towards over estimating $H$ for any series with less than 7,000 observations. The bias was very serious in the short series. For series longer than about 9,000 observations the estimator exhibited a small amount of bias towards underestimating $H$. 
(a) $\mathrm{H}$ Est and $\mathrm{Cl}$ BoxPer $\mathrm{H}=0.60$

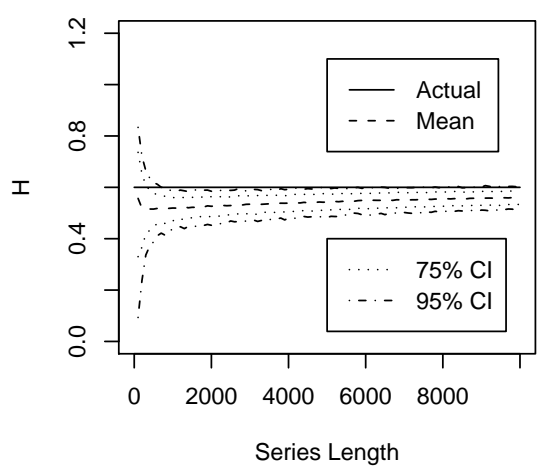

(c) $\mathrm{H}$ Est and $\mathrm{Cl}$ BoxPer $\mathrm{H}=0.90$

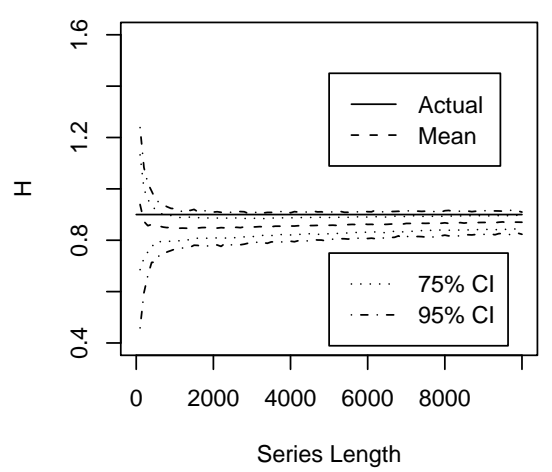

(b) $\mathrm{H}$ Est and $\mathrm{Cl}$ Diffvar $\mathrm{H}=0.60$

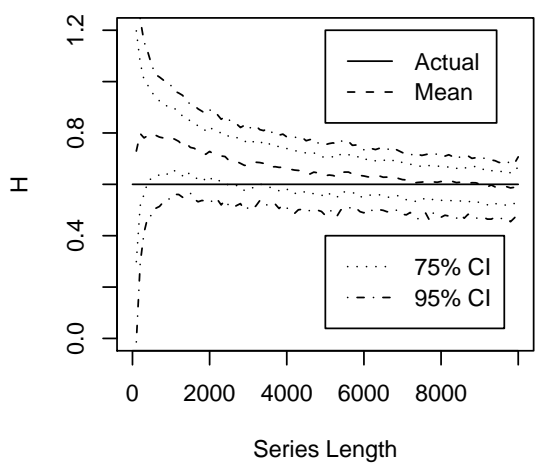

(d) H Est and Cl Diffvar $\mathrm{H}=0.90$

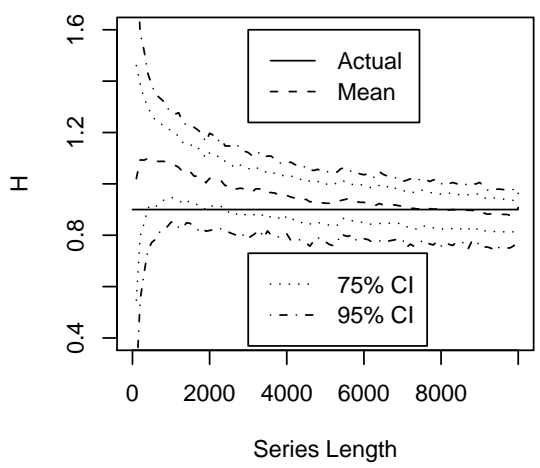

Figure 2. Empirical confidence intervals for the $H$ estimates with $H=0.60$ and $H=0.90$; (a) and (c) boxed periodogram method, (b) and (d) differenced variance estimator.

The results for the Higuchi (1988) estimator are presented in Figures (3) (a) and (c). It was biased towards underestimating $H$ but the magnitude of the bias appeared relatively independent of $H$. The width of the confidence interval of the estimate increased with increasing $H$.

The results for the Peng et al. (1994) estimator are presented in Figures (3) (b) and (d). It was biased toward under estimating $H$ in the series lengths we investigated. This bias appeared to be independent of $H$ but greater in short series.

The results for the R/S estimator are presented in Figures (4) (b) and (d). The R/S estimator exhibited three problems; it was biased upwards when $H$ was low, it was biased downwards when $H$ was high, and the confidence interval of the estimate did not decrease with increasing series length once the series reached about 1000 observations.

The results for the Whittle estimator are presented in Figures (5) (a) and (c). Compared to the other nine estimators implemented in fSeries the Whittle estimator was remarkable for its narrow confidence interval.

The results for the wavelet estimator are presented in Figures (5) (b) and (d). It was unbiased for all $H$ values at series lengths over 4,100 data points. The bias present in series shorter than 4,100 data points was very small. The availability of a new octave can be seen in Figures (5) (b) and (d) with each doubling of the series length. New octaves resulted in a series of steps in the reduction of the confidence interval of the estimate with increasing series length. The estimator had constant variance when the number of 
(a) $\mathrm{H}$ Est and $\mathrm{Cl}$ Higuchi $\mathrm{H}=0.60$

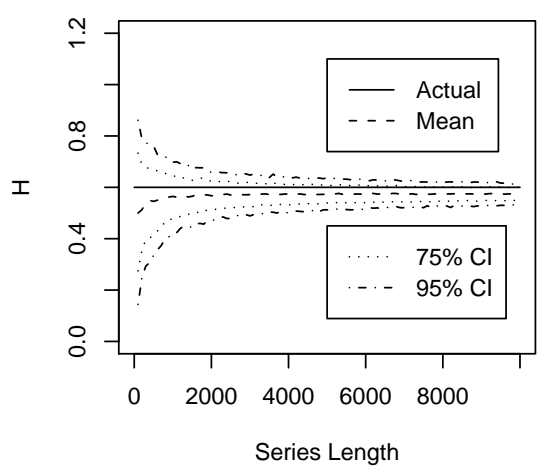

(c) H Est and $\mathrm{Cl}$ Higuchi $\mathrm{H}=0.90$

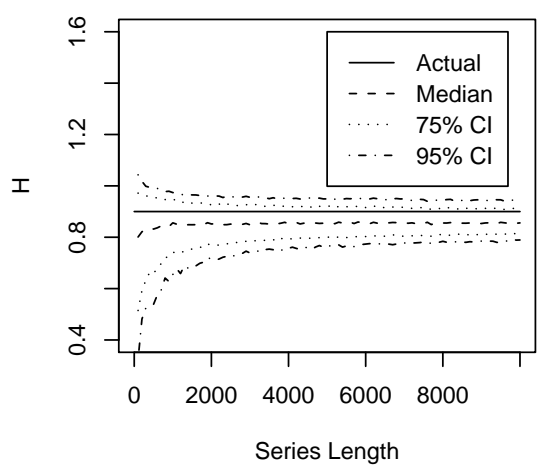

(b) $\mathrm{H}$ Est and $\mathrm{Cl}$ Peng $\mathrm{H}=0.60$

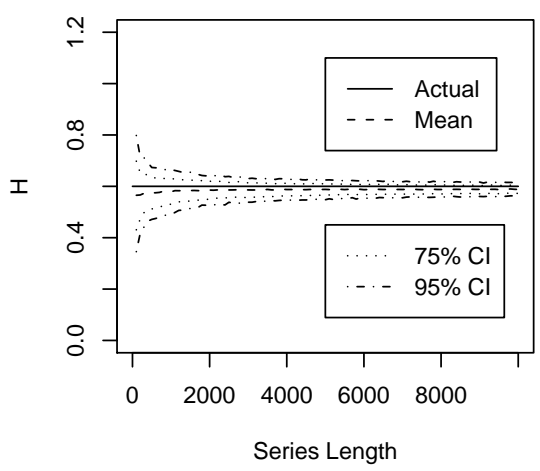

(d) $\mathrm{H}$ Est and $\mathrm{Cl}$ Peng $\mathrm{H}=0.90$

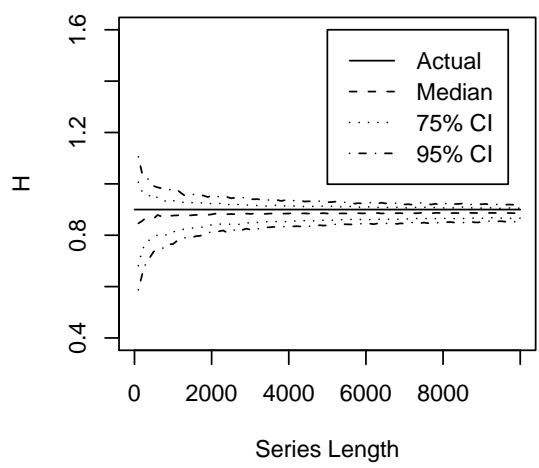

Figure 3. Empirical confidence intervals for the $H$ estimates with $H=0.60$ and $H=0.90$; (a) and (c) Higuchi estimator, (b) and (d) Peng estimator.

octaves was constant.

The results for the GPH estimator are presented in Figures (6)(a) and (c). It exhibited a very small amount of bias towards overestimating $d$ at all series lengths examined. It had a very wide confidence interval which narrowed slowly as the series length increased.

The results for the Haslett-Raftery estimator are presented in Figures (6)(b) and (d). The Haslett-Raftery did not report estimates of $d$ less than zero $(H<0.5)$. Hence for low $d$ and short series the distribution was truncated on the low side at $d=0$ or $H=0.5$ as in Figure (6) (a). The Haslett-Raftery estimator was an excellent estimator with only small amounts of bias in the short series and had a narrow confidence interval.

\section{CONCLUSIONS}

Of the twelve estimators examined here the Whittle and Haslett-Raftery estimators performed the best on simulated series. If we require the estimators to be unbiased and with a 95 percent confidence interval width of less than $0.1 \mathrm{H}$ or $d$ units, then for series with less than 4,000 data points they were the only two estimators worth considering. It should be noted that these estimators did not meet these criteria until the series lengths exceeded 700 and 1000 data points respectively. For series with 4,000 or more data points, the Peng estimator gave acceptable performance. For series with more than 7,000 data points the periodogram estimator was a worthwhile choice. For series with more than 8,200 data points the wavelet 
(a) H Est and $\mathrm{Cl}$ Per. $\mathrm{H}=\mathbf{0 . 6 0}$

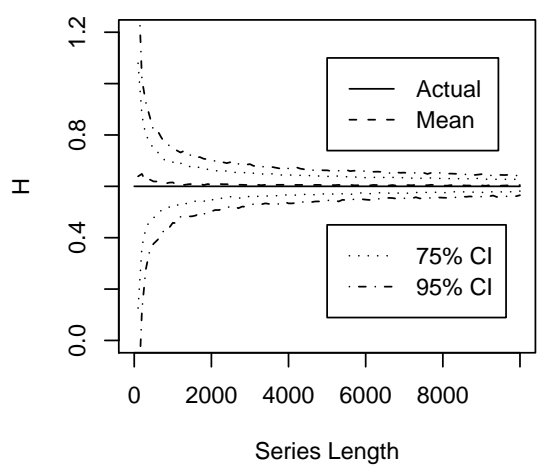

(c) H Est and $\mathrm{Cl}$ Per. $\mathrm{H}=0.90$

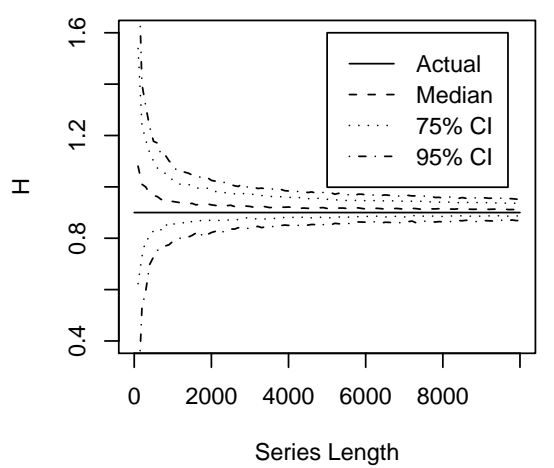

(b) $\mathrm{H}$ Est and $\mathrm{CI} \mathrm{R} / \mathrm{S} \mathrm{H}=0.60$

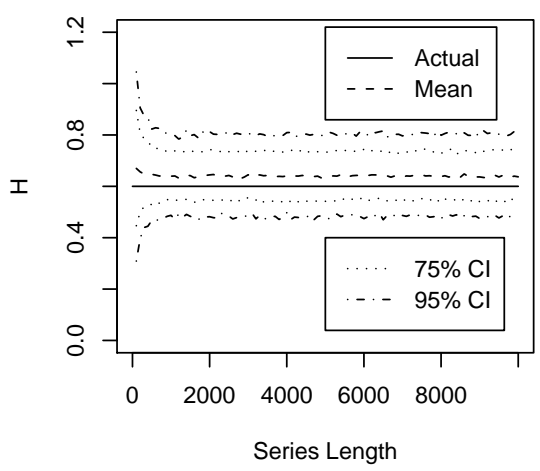

(d) H Est and $\mathrm{Cl}$ R/S H=0.90

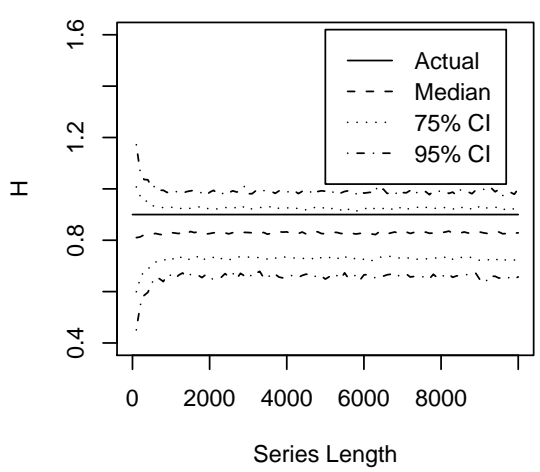

Figure 4. Empirical confidence intervals for the $H$ estimates with $H=0.60$ and $H=0.90$; (a) and (c) periodogram estimator, (b) and (d) R/S estimator.

estimator became a viable estimator. The remaining seven estimators did not give acceptable performance at any series lengths examined and are not recommended.

\section{REFERENCES}

Beran, J. (1994). Statistics for Long Memory Processes. Chapman \& Hall/CRC Press.

Fraley, C., F. Leisch, M. Maechler, V. Reisen, and A. Lemonte (2006). fracdiff: Fractionally differenced ARIMA aka ARFIMA $(p, d, q)$ models. R package version 1.3-0.

Geweke, J. and S. Porter-Hudak (1983). The estimation and application of long memory time series models. Journal of Time Series Analysis 4, 221-237.

Granger, C. W. J. and R. Joyeux (1980). An Introduction to Long-range Time Series Models and Fractional Differencing. Journal of Time Series Analysis 1, 15-30.

Haslett, J. and A. E. Raftery (1989). Space-time Modelling with Long-memory Dependence: Assessing Ireland's Wind Power Resource (with Discussion). Applied Statistics 38(1), 1-50.

Higuchi, T. (1988). Approach to an Irregular Time Series on the Basis of Fractal Theory. Physica D 31, 277-283.

Hosking, J. R. M. (1981). Fractional Differencing. Biometrika 68(1), 165-176. 
(a) H Est and $\mathrm{Cl}$ Whittle $\mathrm{H}=0.65$

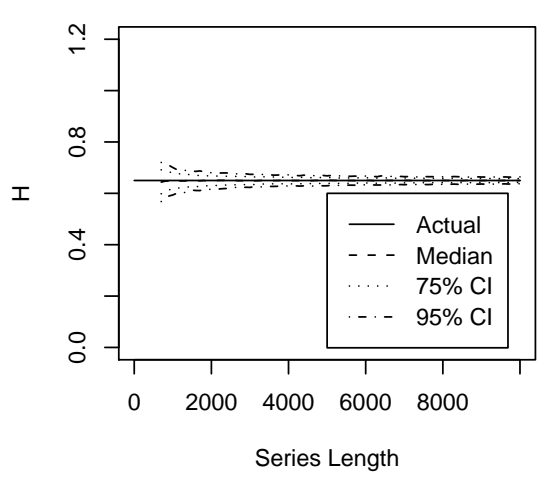

(c) H Est and $\mathrm{Cl}$ Whittle $\mathrm{H}=0.90$

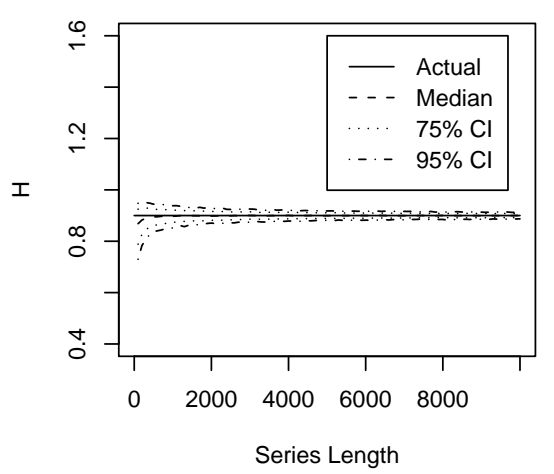

(b) H Est and $\mathrm{Cl}$ Wavelet $\mathrm{H}=0.65$

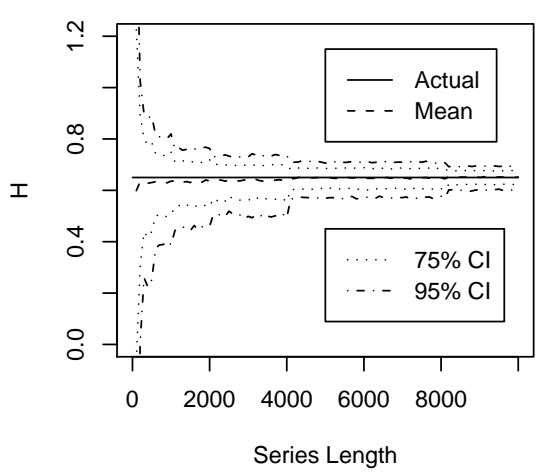

(d) $\mathrm{H}$ Est and $\mathrm{Cl}$ Wavelet $\mathrm{H}=0.90$

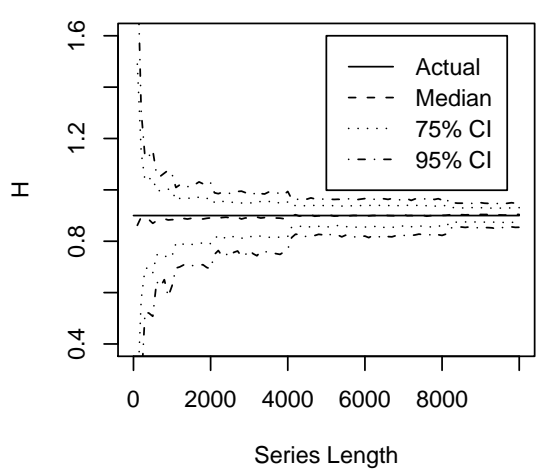

Figure 5. Empirical confidence intervals for the $H$ estimates with $H=0.65$ and $H=0.90$; (a) and (c) Whittle estimator, (b) and (d) wavelet estimator.

Jeong, H.-D. J., J.-S. R. Lee, D. McNickle, and K. Pawlikowski (2007). Comparison of various estimators in simulated FGN. Simulation and Modelling Practice and Theory 15, 1173-1191.

Mandelbrot, B. B. and J. W. van Ness (1968). Fractional Brownian Motions, Fractional Noises and Applications. SIAM Review 10(4), 422-437.

Palma, W. (2007). Long-Memory Time Series Theory and Methods. Wiley-Interscience.

Peng, C. K., S. V. Buldyrev, M. Simons, H. E. Stanley, and A. L. Goldberger (1994). Mosaic organization of DNA nucleotides. Physical Review E 49, 1685-1689.

R Development Core Team (2005). R: A language and environment for statistical computing. Vienna, Austria: R Foundation for Statistical Computing. ISBN 3-900051-07-0.

Robinson, P. M. (2003). Time Series with Long Memory. Oxford University Press.

Taqqu, M., V. Teverovsky, and W. Willinger (1995). Estimators for long-range dependence: an empirical study. Fractals 3(4), 785-798.

Wuertz, D. (2005). fSeries: Financial Software Collection. R package version 220.10063. 
W. Rea et al., The empirical properties of some...

(a) H Est and $\mathrm{Cl} \mathrm{GPH} \mathrm{d=0.10}$

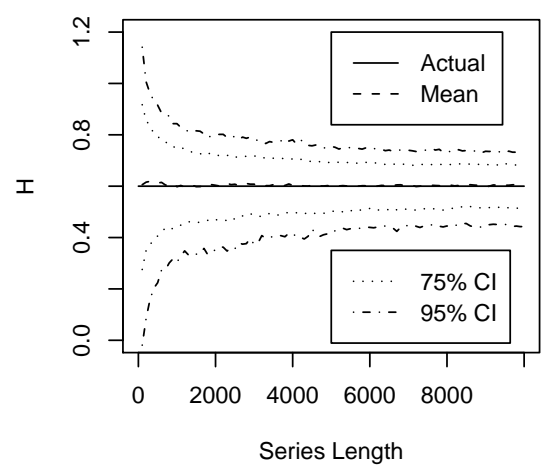

(c) H Est and $\mathrm{Cl} \mathrm{GPH} \mathrm{d=0.40}$

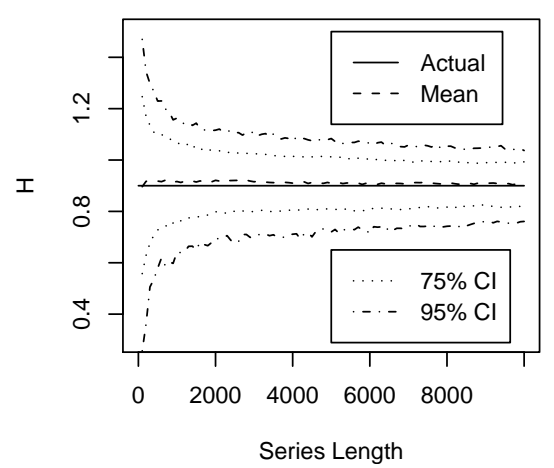

(b) H Est and Cl Haslett-Raftery $d=0.10$

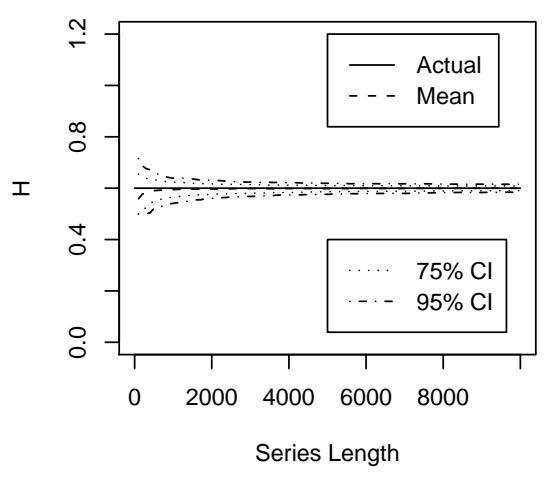

(d) H Est and Cl Haslett-Raftery $\mathrm{d}=0.40$

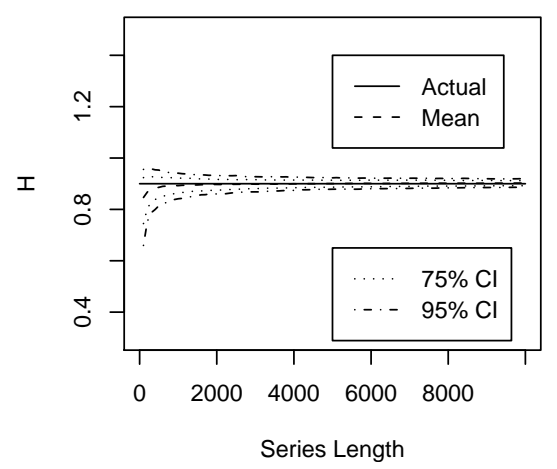

Figure 6. Empirical confidence intervals for the $H$ estimates with $d=0.10(H=0.60)$ and $d=0.40$ $(H=0.90)$; (a) and (c) GPH estimator, (b) and (d) Haslett-Raftery estimator. 